\title{
Study of Factors Associated with the Age of Natural Menopause in Menopausal Women Aged 30 to 80 Years from the Keur Massar Health District in 2015 (Senegal)
}

\author{
Fatoumata Binetou Diongue*, Adama Faye, Khadim Niang, Jean Augustin Diégane Tine, \\ Amadou Ibra Diallo, Rodrigue Magloire Eyema, Anta Tal-Dia
}

Health and Development Institute, Cheikh Anta Diop University of Dakar, Dakar, Senegal

Email: *fatima86d@hotmail.fr

How to cite this paper: Diongue, F.B., Faye, A., Niang, K., Tine, J.A.D., Diallo, A.I., Eyema, R.M. and Tal-Dia, A. (2020) Study of Factors Associated with the Age of Natural Menopause in Menopausal Women Aged 30 to 80 Years from the Keur Massar Health District in 2015 (Senegal). Health, $12,1333-1344$.

https://doi.org/10.4236/health.2020.1210095

Received: May 20, 2020

Accepted: October 10, 2020

Published: October 13, 2020

Copyright $\odot 2020$ by author(s) and Scientific Research Publishing Inc. This work is licensed under the Creative Commons Attribution International License (CC BY 4.0).

http://creativecommons.org/licenses/by/4.0/

\begin{abstract}
Introduction: Menopause is a biological phenomenon that occurs at a more or less early or late age in the life of every woman. The general objective is to study the factors associated with the age of natural menopause in menopausal women aged between 30 and 80 years who have gone through down Keur Massar health district in 2015. Methodology: This was an observational, cross-sectional, descriptive and analytical study conducted in Keur Massar health district from December $1^{\text {st }}$ to $10^{\text {th }}, 2015$. Two-stage cluster sampling was conducted. Data were collected during an individual interview at home after consent. They covered socio-economic conditions, health and nutrition status, gynecological-obstetrical history, and lifestyle. A multivariate analysis was performed using the Cox model with a 5\% risk of alpha error. Results: A total of 627 postmenopausal women were surveyed. Mean age of these women was 54.5 years ( \pm 8.6 years). A proportion of $72.1 \%$ of the women was married and $46.7 \%$ was in school. Mean age of natural menopause was 47.1 years ( \pm 5.03 years). The median age of natural menopause was 47 years. Factors associated with the age of natural menopause were high parity of more than 4 children (Hra $=0.35[0.13-0.92])$, the existence of frequent childhood illnesses $(\mathrm{Hra}=1.72[1.13-2.64])$, use of the intra-uterine device-IUD—as a modern contraceptive method $(\mathrm{HRa}=0.39[0.24-0.63])$, sibling size $(\mathrm{HRa}=$ $0.85[0.78-0.93])$, family size $(\mathrm{HRa}=1.03[1.01-1.06])$, and polygamy regime $(\mathrm{HRa}=0.56[0.37-0.85])$. Conclusion: Many factors in childhood as well as during the period of female genital activity can impact the age of natural menopause. It is then important to improve the health status of women, children and the standard of living of the population.
\end{abstract}




\section{Keywords}

Age Menopause, Associated Factors, Survival, Senegal

\section{Introduction}

According to the World Health Organization (WHO), menopause is defined as a permanent cessation of menstruation resulting from a loss of ovarian follicular activity [1]. This definition is primarily retrospective, since it is based on a 12-month period of consecutive amenorrhea with no obvious physiological or pathological cause [2]. It is a natural phenomenon [1]. Natural menopause usually occurs between the ages of 45 and 55 years. It can be early or late. The steroid hormone deficiency that marks menopause can influence morbidity and mortality by cause during the rest of life. The categories of diseases that are most affected are cardiovascular diseases and malignant tumors [3]. The risk of endometrial cancer increases with age at menopause [4]. Early menopause contributes independently to the risk of post-menopausal fracture [5] and the development of multi-morbidity [6].

The age of menopause depends on the number of follicles in the ovaries. This number begins to decrease at the end of fetal life, and the follicular capital is practically exhausted after menopause [3]. This age of natural menopause varies considerably from one country to another country. In the United States of America, out of a sample of 25,499 women, approximately $65 \%$ are menopausal at age 50 or older [5]. In 2009, Velez et al. [7] reported a median age of 50 years in Latin America and the Caribbean. In Japan the median age of menopause was 52.1 years in 2012 [8], in Europe 54 years [9], in Poland 51.2 years [10]. In Korea a study conducted over the period 2001 to 2014 showed a median and average age of 49.3 years [11]. In Burkina Faso in 2014, an average age of 47.1 years was found [12] and in Senegal 47.8 years in 2006 [13].

Several factors can influence the age of menopause. Socioeconomic difficulties in childhood and adulthood are associated with the age of natural menopause [12]. Vélez et al. [7] in Latin America found that the proportion of women with early menopause was higher among housewives, the nulliparous. Current smoking, low education level, single and unemployed status, and low or moderate physical activity are significantly independently associated with early menopause, while oral contraceptive use, multiparity and good nutrition during childhood are associated with late menopause [9] [10] [11] [14].

Few studies on the age of onset of natural menopause and its determinants are conducted at the population level in Africa and Senegal. This explains the lack of knowledge of the period of occurrence of natural menopause and associated factors, hence the need for a baseline study on natural menopause in Senegal. The general objective is to study the factors associated with the age of natural menopause among menopausal women aged 30 to 80 years who have lived in the 
Keur Massar health district in 2015.

\section{Method}

A quantitative estimate is chosen for this study. The study was observational, cross-sectional, descriptive and analytical over the period from December $1^{\text {st }}$ to $10^{\text {th }}, 2015$. The study population was made up of women aged between 30 and 80 years who had undergone menopause and were living in Keur Massar health district in 2015. This age group is explained by the fact that menopause is documented in Senegal to occur between 28 to 65 age group [13]. The threshold of 80 years was set in order to reduce memory bias. The study took place in the region of Dakar, the capital of Senegal, and more precisely in the suburb of Keur Massar in its health district located $17 \mathrm{~km}$ far from Dakar.

Women with amenorrhea for more than 12 months in the absence of contraception, breastfeeding, hormonal treatment or any other artificial method of non-procreation are included. All women absent at home during the survey after two visits by the interviewer as well as those refusing to sign the informed consent and those residing in the District's area of responsibility for less than 6 months are not included.

The sample represents approximately 600 women. This value is calculated from the Schwartz [15] formula $\left(N=\left(\varepsilon \alpha^{2} \cdot p \cdot q\right) / i^{2}\right)$ with a precision of $5 \%$, a cluster effect of 1.5 and a non-response effect of $2 \%$. This sample was divided into 30 clusters of 20 women. A two-stage cluster survey was conducted. At the first stage, the neighbourhoods in the areas of responsibility are identified. In the second stage, eligible households are selected and all women meeting the criteria are selected. The choice of the first household is made by random selection using the Pen method (stand in the middle of the neighbourhood and choose a given direction randomly) [16]. Then the following households are determined using a sampling step equal to 2 .

The collection is done by direct interview of the women, in their own homes. The information is collected using a questionnaire. The questionnaire is based on a review of validated questionnaires already established for this type of study. It concerns sociodemographic characteristics, behavioural factors, biological factors and environmental factors (previous living conditions, woman's income). Nutritional status was estimated by assessing the satisfaction of food requirements. Questions were asked whether or not there were difficulties in meeting food needs. The socio-economic level was assessed on the basis of household goods. A rating of type 1 and type 2 goods resulted in an accumulation of goods score. The score made it possible to create three classes corresponding to the levels (high, medium, low) of economic well-being. In fact, households were assigned scores based on the number and type of durable goods owned, which goods were air conditioner, television, telephone, refrigerator, radio and also include dwelling characteristics as the source of supply of drinking water, energy for lighting, cooking, flooring material and means of transport. 
The collected data are entered using the EPI INFO version 2000 software and the analysis carried out with the $\mathrm{R}$ software. The univariate analysis made it possible to determine the position or dispersion parameters of the quantitative (mean, standard deviation, median, extremes) and qualitative (absolute and relative frequencies) variables. Overall survival was determined by the Kaplan Meier method [17]. The Logrank test was used to assess the relationship between two variables and to plot survival curves according to the variables.

The multivariate analysis is done by modelling with the Cox model. A risk of alpha error $(\alpha)$ of $5 \%$ is taken. All variables that had a $p$-value of less than 0.25 were included in the model. Then following the step-by-step top-down strategy, the variables whose $p$-values are not significant are excluded from the model. The final model was validated by the Schoenfild test [18]. This multivariate analysis made it possible to determine the adjusted Hazard Ratio (HR).

\section{Results}

\subsection{Characteristics of Women}

The study involved a total of 627 postmenopausal women. The average age of the women was 54.5 years with a standard deviation of 8.6 years. All women knew their age at the onset of menopause. The mean age of natural menopause was 47.1 years with a standard deviation of 5.03 years. The median age was 47 years. Married women accounted for $72.1 \%, 56 \%$ of whom were on a monogamous status. They were enrolled in school in $46.7 \%$ (Table 1).

\subsection{Characteristics of Women in Childhood}

A proportion of $44.7 \%$ of the women's parents lived in the city and $46.1 \%$ in the village. The average family size was $18.03( \pm 9.7)$. It ranged from 2 to 52 persons with a median size of 15 members.

A proportion of $98.4 \%$ of women's fathers were gainfully employed, and the parents of women with high socio-economic status were $59.2 \%$ and those with low socio-economic status were $18.6 \%$.

\subsection{Characteristics of Women in the Period of Genital Activity}

During the period of genital activity, $44 \%$ of the women had a paid activity. A proportion of $22.5 \%$ of women had difficulty meeting their food needs. A proportion of $84.5 \%$ of women had means to consult in a public hospital and $71.9 \%$ owned their living quarters (Table 1).

\subsection{Lifestyle Characteristics}

Women in regular physical activity of at least 30 minutes a day accounted for $27.3 \%$; $8 \%$ consumed alcoholic beverages and $2.4 \%$ smoked. Women whose spouses smoked accounted for $20.6 \%$. Thus, the proportion of women who smoked actively was $2.4 \%$ and that of women who smoked passively was $19.6 \%$ (Table 2). 
Table 1. Distribution of postmenopausal women by socio-demographic and economic characteristics in childhood and period of genital activity $(\mathrm{n}=627)$.

\begin{tabular}{|c|c|c|}
\hline & Absolute frequencies (n) & Relative Frequencies (\%) \\
\hline \multicolumn{3}{|c|}{ Socio-demographic characteristics } \\
\hline \multicolumn{3}{|l|}{ Marital status } \\
\hline Married & 452 & 72.1 \\
\hline Widow & 104 & 16.6 \\
\hline Divorced & 31 & 4.9 \\
\hline Single & 29 & 4.6 \\
\hline Separated & 11 & 1.8 \\
\hline \multicolumn{3}{|l|}{ Schooling } \\
\hline Yes & 293 & 46.7 \\
\hline No & 334 & 53.3 \\
\hline \multicolumn{3}{|l|}{ Religion } \\
\hline Muslims & 554 & 88.3 \\
\hline Christians & 70 & 11.2 \\
\hline Traditional beliefs & 3 & 0.5 \\
\hline \multicolumn{3}{|l|}{ Ethnic group } \\
\hline Ouolof & 246 & 39.2 \\
\hline Pular & 105 & 16.8 \\
\hline Serere & 102 & 16.3 \\
\hline Diola & 64 & 10.2 \\
\hline Others & 110 & 17.5 \\
\hline \multicolumn{3}{|c|}{ Economic characteristics during childhood } \\
\hline \multicolumn{3}{|l|}{ Habitat area } \\
\hline City & 280 & 44.7 \\
\hline Village & 289 & 46.1 \\
\hline Village then town & 47 & 7.5 \\
\hline City then village & 10 & 1.6 \\
\hline Unspecified & 1 & 0.2 \\
\hline \multicolumn{3}{|c|}{ Type of family environment experienced } \\
\hline Siring parents & 407 & 64.9 \\
\hline Paternal parents & 123 & 19.6 \\
\hline Maternal parents & 64 & 10.2 \\
\hline Family friend & 5 & 0.8 \\
\hline Others & 3 & 0.5 \\
\hline Unspecified & 25 & 4.0 \\
\hline \multicolumn{3}{|c|}{ Paid activity of the father } \\
\hline Yes & 617 & 98.4 \\
\hline No & 10 & 1.6 \\
\hline \multicolumn{3}{|c|}{ Difficulty in meeting food needs } \\
\hline Yes & 158 & 25.2 \\
\hline No & 469 & 74.8 \\
\hline \multicolumn{3}{|l|}{ Difficult childhood } \\
\hline Yes & 65 & 10.4 \\
\hline No & 562 & 89.6 \\
\hline \multicolumn{3}{|c|}{ Socio-economic level } \\
\hline High & 371 & 59.2 \\
\hline Medium & 139 & 22.2 \\
\hline Low & 117 & 18.6 \\
\hline
\end{tabular}


Continued

Economic characteristics during the period of genital activity

\begin{tabular}{|c|c|c|}
\hline \multicolumn{3}{|c|}{ Women with a paid activity } \\
\hline Yes & 276 & 44.0 \\
\hline No & 351 & 56.0 \\
\hline \multicolumn{3}{|c|}{ Meeting basic needs } \\
\hline Yes & 567 & 90.4 \\
\hline No & 60 & 9.6 \\
\hline \multicolumn{3}{|c|}{ Difficulty in ensuing food needs } \\
\hline Yes & 141 & 22.5 \\
\hline No & 486 & 77.5 \\
\hline \multicolumn{3}{|c|}{ Judgement of one's own economic situation } \\
\hline Good & 203 & 32.4 \\
\hline Medium & 362 & 57.7 \\
\hline Poor & 53 & 8.5 \\
\hline Unspecified & 9 & 1.4 \\
\hline \multicolumn{3}{|c|}{ Means of consultation in public hospitals } \\
\hline Yes & 530 & 84.5 \\
\hline No & 94 & 15.0 \\
\hline Unspecified & 3 & 0.5 \\
\hline \multicolumn{3}{|c|}{ Housing owned or rented } \\
\hline Owner & 451 & 71.9 \\
\hline For rent & 172 & 27.4 \\
\hline Unspecified & 4 & 0.6 \\
\hline \multicolumn{3}{|c|}{ Socio-economic level } \\
\hline High & 373 & 59.5 \\
\hline Medium & 188 & 29.9 \\
\hline Low & 66 & 10.6 \\
\hline
\end{tabular}

\subsection{Characteristics Related to Women's Medical History and Genital Activity}

A proportion of $29.5 \%$ of the women had common childhood illnesses. Women with regular cycles before the age of 40 represented $72.2 \%$. The average age of menarche was 14.2 years ( \pm 1.7 years). The average length of women's menstrual cycles was 28.3 days $( \pm 2.1)$ and $54 \%$ of women had a regular cycle of 28 days. The average length of women's menstruation was 5.1 days ( \pm 2.2 days). The average number of children was $5.5( \pm 2.6)$ ranging from 0 to 14 children. A total of 21 women $(3.3 \%)$ had no children and $62 \%$ had 5 or more children (Table 2 ).

\subsection{Characteristics Related to the Use of Modern Contraceptives}

A proportion of $22 \%$ or 138 of the women were on oral contraceptives. Women who used other contraceptive methods accounted for $22.5 \%$. A proportion of $7.5 \%$ used the intra-uterine device (IUD); $10.8 \%$ used injectables; $4.6 \%$ used implants and $2.3 \%$ used the natural method (Table 2).

\subsection{Overall Survival and Factors Associated with Natural Menopause Age}

The overall survival from natural menopause, as determined by the Kaplan-Meier 
Table 2. Distribution of women by lifestyle habits, medical history and genital activity $(\mathrm{n}=627)$.

\begin{tabular}{|c|c|c|}
\hline & Absolute Frequencies ( $n$ ) & Relative Frequencies (\%) \\
\hline \multicolumn{3}{|c|}{ Characteristics related to women's lifestyles } \\
\hline \multicolumn{3}{|c|}{ Regular physical activity of at least $30 \mathrm{~min} /$ day } \\
\hline Yes & 171 & 27.3 \\
\hline No & 456 & 72.7 \\
\hline \multicolumn{3}{|c|}{ Consumption of alcoholic beverages } \\
\hline Yes & 52 & 8.0 \\
\hline No & 550 & 88.0 \\
\hline Unspecified & 25 & 4.0 \\
\hline \multicolumn{3}{|l|}{ Tobacco consumption } \\
\hline Yes & 15 & 2.4 \\
\hline No & 567 & 90.4 \\
\hline Unspecified & 45 & 7.2 \\
\hline \multicolumn{3}{|l|}{ Type of smoking } \\
\hline Old smoking & 04 & 26.7 \\
\hline Current smoking & 07 & 46.6 \\
\hline Unspecified & 04 & 26.7 \\
\hline \multicolumn{3}{|l|}{ Partner's tobacco consumption } \\
\hline Yes & 129 & 20.6 \\
\hline No & 479 & 76.4 \\
\hline Unspecified & 19 & 3.0 \\
\hline \multicolumn{3}{|l|}{ Smoking mode } \\
\hline Active smoking & 15 & 2.4 \\
\hline Passive smoking & 123 & 19.6 \\
\hline No smoking & 448 & 71.5 \\
\hline Unspecified & 41 & 6.5 \\
\hline \multicolumn{3}{|l|}{ Breastfeeding method used } \\
\hline Exclusive breastfeeding (AME) & 352 & 58.1 \\
\hline Artificial feeding & 31 & 5.1 \\
\hline Mixed feeding & 214 & 35.3 \\
\hline Unspecified & 9 & 1.5 \\
\hline \multicolumn{3}{|c|}{ Characteristics related to women's medical records and genital activity } \\
\hline \multicolumn{3}{|l|}{ Common childhood illnesses } \\
\hline Yes & 185 & 29.5 \\
\hline No & 379 & 60.4 \\
\hline Unspecified & 63 & 10.0 \\
\hline \multicolumn{3}{|c|}{ Existence of a history of prior serious disease } \\
\hline Yes & 156 & 24.9 \\
\hline No & 468 & 74.6 \\
\hline Unspecified & 3 & 0.3 \\
\hline \multicolumn{3}{|l|}{ Malnutrition } \\
\hline Yes & 33 & 5.3 \\
\hline No & 594 & 94.7 \\
\hline \multicolumn{3}{|c|}{ Hospitalization during childhood } \\
\hline Yes & 96 & 15.3 \\
\hline No & 522 & 83.3 \\
\hline Unspecified & 9 & 1.4 \\
\hline
\end{tabular}




\section{Continued}

Regularity of mentrual cycles before 40

Yes

453

168

6

Amount of blood during menstruation

Little

Abundant

Unspecified
Unspecified

72.2

26.8

1.0

13.9

53.3

31.9

1.0

Parity

Nulliparous

21

209

3.3

One to four children

392

33.3

Five children and more

Unspecified

\section{Abortions}

Yes

No

Unspecified

Characteristics related to the use of modern family planning methods

Use of oral contraceptives

Yes

No

Unspecified

Use of other contraceptive methods

Yes

No

Unspecified

IUD (Intra-Uterine Device) use

Yes

No

Unspecified

Use of injectables

Yes

No

Unspecified

Use of the natural method

Yes

No

Unspecified

\section{Use of implants}

Yes

No

Unspecified

$\begin{array}{cc}29 & 4.6 \\ 132 & 21.1 \\ 466 & 74.3\end{array}$

method, is shown in Figure 1. The median age of menopause was 47 years, corresponding to $50 \%$ of women who had already gone through menopause.

Multivariate analysis with the Cox model gave the explanatory factors for the age of menopause. Thus high parity of more than 4 children in women reduces 


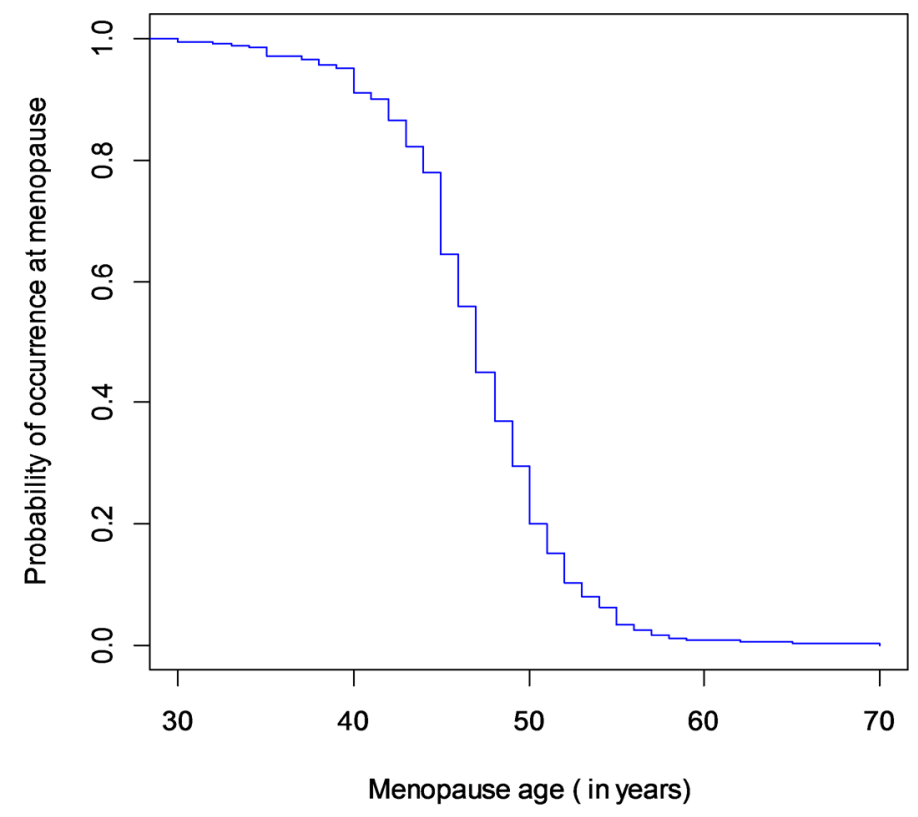

Figure 1. Graphic representation of overall survival for natural menopause age.

the risk of early menopause $(\mathrm{HRa}=0.35[0.13-0.92])$. In the same way, women with older siblings $(\mathrm{HRa}=0.85[0.78-0.93])$ and those on a polygamous status $(\mathrm{HRa}=0.56[0.37-0.85])$ have a lower risk of early menopause. The use of the IUD as a modern contraceptive method is therefore associated with a later age of menopause $(\mathrm{HRa}=0.39[0.24-0.63])$. In addition, women who have lived in large families and those with frequent childhood illnesses have a high risk of earlier menopause with respectively $(\mathrm{HRa}=1.03[1.01-1.06])$ and $(\mathrm{HRa}=1.72$ [1.13 - 2.64]) (Table 3).

\section{Discussion}

Limits of study: our study has a number of limitations that should be noted. Menopause is most often a taboo subject in Senegal societies, and raising such a subject with women can lead to information bias. We carried out a descriptive cross-sectional survey for analytical purposes to be able to identify the factors associated with the age of natural menopause by a multivariate analysis. These associations can be better demonstrated by a cohort study. Our survey came at a time when women had their last period for at least 12 months and menarche for several years. This can be a source of information bias, particularly recall bias. Also, it is possible that women with menopause rather have died at a young age which can underestimate the real links that exist.

This study shows a median age of natural menopause of 47 years. These results are comparable to those found by JC Moreau et al. [13], Faye et al. in Burkina Faso [12], Garrido-Latorre et al. in Mexico [19], Velez et al. [7] in Santiago de Chile, and Kwawukume in Ghana [20]. However, in the USA Gold et al. [14], in Europe Dratva et al. [9], in Japan Toshiyuki et al. [8], in Bridgetown and Sao 
Table 3. Factors associated with age at menopause in multivariate analysis with the Cox model.

\begin{tabular}{lc}
\hline \multicolumn{1}{c}{ Characteristics of women } & Adjusted HR (CI to 95\%) \\
\hline Marital status & 1 \\
Married & $3.46[0.80-14.91]$ \\
Unmarried & $2.26[0.87-5.88]$ \\
Widower & \\
Matrimonial regime of women married & 1 \\
Monogamy & $0.56[0.37-0.85]$ \\
Polygamous & $0.85[0.78-0.93]$ \\
Size of the siblings & $1.03[1.01-1.06]$ \\
Size of the family & \\
Existence of frequent diseases during childhood & 1 \\
No & $1.72[1.13-2.64]$ \\
Yes & \\
Use of the inta-uterine device (IUD) & 1 \\
No & $0.39[0.24-0.63]$ \\
Yes & \\
Parity (Number of children per woman) & 1 \\
Zero to one & \\
Two to four & \\
Five and more & $0.43[0.16-1.16]$ \\
\hline
\end{tabular}

Paulo (Brazil) in Latin America Velez et al. [7], in Korea Park et al. [11] found median menopausal ages of 51.4, 54, 52.1, 50, and 49.3 years, respectively. This variation reflects the differences between countries with high and low living standards.

Factors associated with age at menopause found in this study represent marital status, siblings, common childhood illnesses, use of the intra-uterine device (IUD) as a modern contraceptive method, and high parity.

In our fitted model, high parity is associated with late menopause. Indeed, women with five or more children have an advanced age of menopause compared to those who are nulliparous or have one child. However, such an effect was not found in women with two to four children. Faye et al. found similar results in Burkina Faso [12], as did Garrido-Latorre in Mexico [19] and Kaczmarek in Poland [10]. Christine et al. in her multivariate model also found higher parity associated with a lower risk of early menopause [21]. The number of children per woman can influence the age of menopause. Park et al. [11] in Korea found that women who gave birth were less likely to experience early menopause.

In our study, the age of menopause appears to be higher in women on a polygamous status than in those on a monogamous status. This can be explained by the fact that in our Senegalese context, women in polygamy tend to have many children because of rivalry between co-wives contributing to the reduction of follicular loss. Thus the extinction of follicular activity will occur at an advanced age, hence the menopause at a late age. Park et al. [11] in Korea found that the 
absence of a partner made the earlier onset of natural menopause more likely.

Our study also found a relationship between sibling size and family size with age at menopause. Larger siblings would decrease the risk of early menopause while larger family size would increase this risk.

Another factor that has been found to influence the age of menopause is the frequent illnesses in childhood. Women with episodes of childhood illness are at higher risk of menopause at an earlier age than others. Kaczmarek found the same results in Poland [10]: women with a good and fairly good childhood health status are less likely to go through menopause before women with a poor health status. Gold [14] in her study judged health status using a history of heart disease and found that women with a history of heart disease were more likely to go through menopause before those without a history of heart disease.

Our study showed a reduced risk of early menopause among IUD users compared to non-IUD users. However, Anna and Nick [22] found an association between contraceptive pill use and later menopausal age.

\section{Conclusion}

Factors that can influence the age of menopause arise in both childhood and adulthood. Our study found that these factors are related to the environment, lifestyle habits, and particularly female genital activity. Thus it is important to improve the health status of women, children and the standard of living of the population.

\section{Conflicts of Interest}

The authors declare no conflicts of interest regarding the publication of this paper.

\section{References}

[1] World Health Organisation (WHO) (1996) Research on Menopause: Review of the 1990s: Report of a WHO Scientific Panel. Technical Report Series 866. WHO Publications, Geneva.

[2] Drapier-Faure, E. and Jamin, C. (2003) La ménopause. Doin Editions.

[3] Chapter III: Menopause. http://www.medecine.ups-tlse.fr/dcem3/module05/55_poly_1_menopause.pdf

[4] Wu, Y., et al. (2019) Age at Menopause and Risk of Developing Endometrial Cancer: A Meta-Analysis. BioMed Research International, 2019, Article ID: 8584130. https://doi.org/10.1155/2019/8584130

[5] Sullivan, S.D., et al. (2017) Age of Menopause and Fracture Risk in Post-Menopausal Women Randomized to Calcium + Vitamin D, Hormone Therapy, or the Combination: Results from the Women's Health Initiative Clinical Trials. Menopause, 24, 371-378. https://doi.org/10.1097/GME.0000000000000775

[6] Xu, X., Jones, M. and Mishra, G.D. (2020) Age at Natural Menopause and Development of Chronic Conditions and Multimorbidity: Results from an Australian Prospective Cohort. Human Reproduction, 35, 203-211.

https://doi.org/10.1093/humrep/dez259 
[7] Velez, M.P., et al. (2010) Life Course Socioeconomic Adversity and Age at Natural Menopause in Women from Latin America and the Caribbean. Journal of the North American Menopause Society, 17, 552-559. https://doi.org/10.1097/gme.0b013e3181ceca7a

[8] Toshiyuki, Y., et al. (2012) Factors Associated with Premature Ovarian Failure, Early Menopause and Earlier Onset of Menopause in Japanese Women. The European Menopause Journal, 72, 249-255. https://doi.org/10.1016/j.maturitas.2012.04.002

[9] Dratva, J., Gomez, R.F., Schindler, C., et al. (2009) Is Age at Menopause Increasing across Europe? Results on Age at Menopause and Determinants from Two Population-Based Studies. Menopause, 16, 385-394.

https://doi.org/10.1097/gme.0b013e31818aefef

[10] Kaczmarek, M. (2007) The Timing of Natural Menopause in Poland and Associated Factors. The European Menopause Journal, Maturitas, 57, 139-153.

https://doi.org/10.1016/j.maturitas.2006.12.001

[11] Park, C.Y., Lim, J.Y. and Park, H.Y. (2018) Age at Natural Menopause in Koreans: Secular Trends and Influences Thereon. Menopause, 25, 423-429.

https://doi.org/10.1097/GME.0000000000001019

[12] Faye, A., Nikiema, C. and Tal-Dia, A. (2017) Socioeconomic Adversities during Life Course and Menopause Onset in a Developing Country. Epidemiology (Sunnyvale), 7, 319. https://doi.org/10.4172/2161-1165.1000319

[13] Moreau, J.C., Cissé, C.T., Diouf, A.A., Kane-Guèye, S.M., Dieng, T. and Guèye-Dièye, A. (2015) Epidemiology, Experience and Management of Menopause in Dakar. CGO-CEFOREP. http://www.saerm-dz.com/upload/File/saerm_7c/4-\%20JP.\%20MOREAU.pdf

[14] Gold, E.B., Bromberger, J., Crawford, S., et al. (2001) Factors Associated with Age at Natural Menopause in a Multiethnic Sample of Midlife Women. American Journal of Epidemiology, 153, 865-874. https://doi.org/10.1093/aje/153.9.865

[15] Schwartz, D. (1963) Méthodes statistiques à l'usage des médecins et biologistes. Edition Flammarion Médecins Sciences, Paris, 291 p.

[16] Helen Keller International (2014) Guide pour la réalisation des enquêtes de couverture post-événement de supplémentation en vitamine $\mathrm{A}$, de déparasitage et de vaccination. Affaire étrangère, Commerce et Développement, Canada. 74, 21.

[17] Altman, D.G. (1992) Analysis of Survival Times. In: Practical Statistics for Medical Research, Chapman and Hall, London, 365-393.

[18] Schoenfeld, D. (1982) Partial Residuals for the Proportional Hazards Regression Model. Biometrika, 69, 239-241. https://doi.org/10.1093/biomet/69.1.239

[19] Garrido-Latorre, F., Lazcano-Ponce, E.C., Lopez-Carrillo, L., et al. (1996) Age of Natural Menopause among Women in Mexico City. International Journal of Gynecology and Obstetrics, 53, 159-166. https://doi.org/10.1016/0020-7292(96)02655-0

[20] Kwawukume, E.Y., Ghosh, T.S. and Wilson, J.B. (1993) Menopausal Age of Ghanaian Women. International Journal of Gynecology \& Obstetrics, 40, 151-155. https://doi.org/10.1016/0020-7292(93)90376-8

[21] Christine, R., et al. (2020) Association of Parity and Breastfeeding with Risk of Early Natural Menopause. JAMA Network Open, Obstetric and Gynecology, 3, e1919615. https://doi.org/10.1001/jamanetworkopen.2019.19615

[22] Fenton, A. and Panay, N. (2015) What Influences the Age of Menopause? Journal Climacteric, 18, 767-768. https://doi.org/10.3109/13697137.2015.1097049 